ECS Transactions, 41 (1) 845-852 (2011)

10.1149/1.3635618 C The Electrochemical Society

\title{
Effect of Graphitic Content on Carbon Supported Catalyst Performance
}

\author{
Anant Patel ${ }^{\mathrm{a}}$, Kateryna Artyushkova ${ }^{\mathrm{a}}$, Plamen Atanassova ${ }^{\mathrm{a}}$, David Harvey ${ }^{\mathrm{b}}$, Monica Dutta ${ }^{\mathrm{b}}$, \\ Vesna Colbow ${ }^{\mathrm{b}}$, and Silvia Wessel ${ }^{\mathrm{b}}$ \\ ${ }^{a}$ Chemical \& Nuclear Engineering Department, UNM Center for Emerging Energy \\ Technologies, University of New Mexico, Albuquerque, NM 87131 \\ ${ }^{\mathrm{b}}$ Ballard Power Systems, 9000 Burnaby, BC V5J 5J8, Canada
}

The effect of graphitic content on carbon supported platinum catalysts was investigated in order to investigate its influence on catalyst performance. Four catalysts of varying surface areas and graphitic content were analyzed using XPS, HREELS, and tested using RDE experiments. The catalysts were also heat treated at $150^{\circ} \mathrm{C}$ and $100 \% \mathrm{RH}$ as means to uniformly age them. The heat treated samples were analyzed using the same methods to determine what changes had occurred due to this aging process. When compared to the BOL catalysts, heat treated catalysts displayed increased graphitic carbon and platinum metallic content, however they also showed depressed catalytic activity. The primary cause is still under investigation, though it is believed to be related to loss of amorphous carbon content.

\section{Introduction}

Increasing economic pressures are restricting the use of platinum in PEMFCs, leading to carbon blacks becoming the predominant support material. Carbon blacks, however, lack the robustness of platinum blacks, impeding the widespread implementation of the power systems. As such, the object of this work is to identify which descriptors of carbon blacks change due to exposure to the PEMFC environment. Carbon blacks are a diverse and fairly complex group of materials, therefore by examining several samples before and after aging, a descriptive understanding of carbonaceous support materials can be determined.

Samples in this work are separated in to four groups: undecorated low, mid-range, and high surface area carbons, variable Pt loadings on low surface area carbon, constant Pt loading each carbon support, and control samples such as HOPG. These groups of will be subjected to electrochemical testing and various characterization techniques in order to quantify the systems.

Catalyst performance and aging studies are being done using a rotating disk electrode (RDE. Comparing the catalysts performance before and after aging provides the means of gauging performance loss. $(1,2)$

Of primary importance to this study is electron energy loss spectroscopy (HREELS). This technique is a powerful tool capable of differentiating between carbon structures, such as graphite, diamond, or amorphous carbons.(3) Graphite is known to be less susceptible to degradation than other forms of carbon, but how this will affect $\mathrm{Pt}$ nanoparticles remains unclear. By fitting the spectra obtained from each sample, the relative percent of graphite content can be determined.

Along with HREELS, X-ray photoelectron spectroscopy (XPS) has been done to analyze the chemical composition of the samples surfaces.(4) Because catalysis is a surface phenomenon, understanding the surface that will be exposed to the fuel cell environment has significant implications.

\section{$\underline{\text { Materials }}$}

\section{Materials and Methods}

In order to understand the effect of the carbon support on catalyst degradation, distinctly different carbon supports, low surface area (LSA), high surface area (HSA), 
and mid-range surface area (MSA) carbons were investigated. High surface area carbons have a larger number of catalyst anchor sites yielding well-dispersed catalyst particles on the support. This level of dispersal is believed to result in increased kinetic performance. However, the large number of surface sites means these carbons will be more susceptible to corrosion, thus altering their performance and level of degradation. In order to stabilize the high surface area carbon, it was heat treated to cause some degree of graphitization, with the resulting material being labeled HSAHT.

\section{Characterization}

X-ray photoelectron spectroscopy (XPS) was performed using Kratos Axis Ultra spectrometer using an $\mathrm{Al} \mathrm{K} \alpha \mathrm{X}$-ray source, with emission voltage of $12 \mathrm{kV}$ and emission current of $20 \mathrm{~mA}$. Both low resolution survey spectra and high resolution $\mathrm{C} 1 \mathrm{~s}$ and $\mathrm{Pt} 4 \mathrm{f}$ spectra were acquired. All XPS data were charge-referenced to aromatic carbon at 284.7 $\mathrm{eV}$.

Electron energy loss spectroscopy (HREELS) was used done using a JEOL 2010F TEM using a field emission gun as the source with a voltage of $200 \mathrm{kV}$, and beam energy of $0.1 \mathrm{kV}$. The unaltered and aged catalysts were investigated using this method. To ensure that the results were accurate, the spectra were acquired at 15 random locations per sample. Finally, highly ordered pyrolytic graphite was used as a reference sample because of its $100 \% \mathrm{sp}^{2}$ bonding nature.

\section{$\underline{\text { Performance Measurements }}$}

All samples were prepared as $4 \mathrm{mg} / \mathrm{ml}$ catalyst inks and tested using a rotating disk electrode (RDE) in a 3-electrode system. The electrodes were rotated at $1600 \mathrm{RPM}$, and were cycled between 0 and $1.2 \mathrm{~V}$ (vs. RHE) at a sweep rate of $5 \mathrm{mV} / \mathrm{s}$. The electrochemical cell used $0.1 \mathrm{M} \mathrm{HClO}_{4}$ as the electrolyte, and oxygen gas as the fuel.

\section{Results and Discussion}

The goal of this work was to relate changes in performance to graphitic content. As such, Electron Energy Loss Spectroscopy (HREELS) and X-ray Photoelectron Spectroscopy (XPS) were chosen to obtain information on graphitic content (degree of crystallinity) and surface oxide content of the carbon supports.

The ability to discriminate between different carbon chemical environments, not just elemental compositions, is one of the primary advantages of XPS in the characterization of carbon corrosion; however, HREELS is the preferred method to quantitatively extract the $\mathrm{sp}^{2}$ fraction of carbon-based materials from carbon K-edge spectrum.

\section{$\underline{\text { XPS results }}$}

Figure 1 shows C 1s XPS spectra for LSAC 50 and HSAC 50 samples along with curve fit. A different ratio of graphitic (blue) peak to the rest of the peak is obvious.
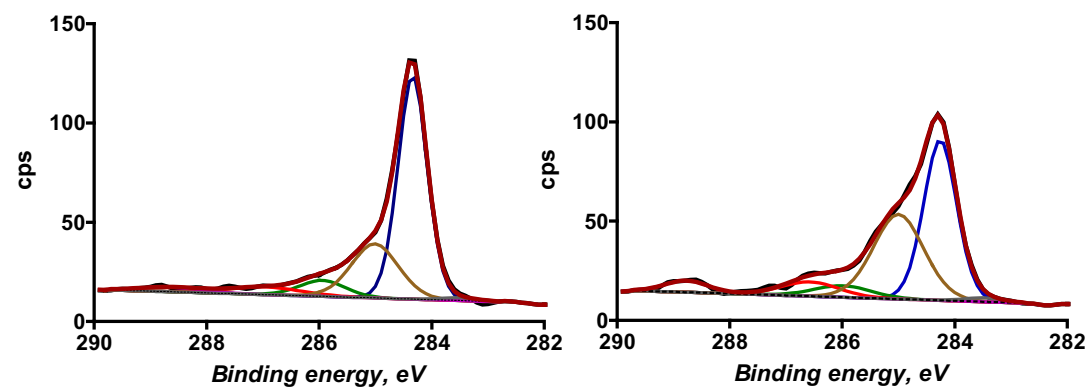

Figure 1. High resolution C 1s spectra for a) LSAC50 and b) MSAC50 samples shown with curve fits used to deconvolute species as identified in Table 1

Table 1 shows results of curve fit of XPS spectra for BOL and HT samples. The values 
shown are the average of 3 areas.

TABLE 1. C speciation as determined from XPS spectra

\begin{tabular}{|c|c|c|c|c|c|c|}
\hline \multirow[t]{2}{*}{ Sample ID } & carbide & C gr & $\mathrm{C}-\mathrm{C}$ & $C^{*}-\mathrm{C}-\mathrm{X}$ & $\begin{array}{c}\mathrm{C}-\mathrm{OH} / \mathrm{C}- \\
\mathrm{OC}\end{array}$ & $\mathbf{C}=\mathbf{O}$ \\
\hline & 283.4 & 284.5 & 285.1 & 285.7 & 286.7 & 289.1 \\
\hline \multicolumn{7}{|c|}{ Beginning of Life } \\
\hline LSAC 50 & 1.2 & 58.6 & 25.4 & 6.1 & 5.7 & 2.9 \\
\hline MSAC 50 & 0.7 & 39.5 & 41.0 & 8.6 & 5.9 & 4.4 \\
\hline HSAC1 50 & 1.5 & 44.1 & 35.8 & 6.9 & 7.0 & 4.7 \\
\hline HSAC HT 50 & 1.0 & 56.7 & 26.4 & 3.8 & 8.6 & 3.5 \\
\hline \multicolumn{7}{|c|}{ After Heat Treatment } \\
\hline LSAC 50 & 2.8 & 56.9 & 21.4 & 6.1 & 9.4 & 3.4 \\
\hline MSAC 50 & 3.6 & 53.0 & 23.0 & 8.1 & 8.7 & 3.6 \\
\hline HSAC1 50 & 2.3 & 54.1 & 23.5 & 5.6 & 11.8 & 2.8 \\
\hline HSAC HT 50 & 2.3 & 51.1 & 25.8 & 6.9 & 9.4 & 4.6 \\
\hline
\end{tabular}

For BOL samples, increasing surface area anti-correlates approximately with graphitic content, with MSAC having the lowest percentage of graphite. Heat treating HSAC brings graphite content to almost the same level as LSAC sample. Samples with lowest graphitic content also have the highest amounts of aliphatic carbons. Additionally, the amount of surface oxides also increases with increasing surface area. Heat treatment of HSAC sample does not change the amount of surface oxides, but rather causes reorganization of graphitic and aliphatic carbons.

Heat treatment of LSAC and HSAC HT almost did not change the amount of graphitic carbon. The largest changes are detected for MSAC where an approximately $30 \%$ increase of graphitic content is observed and HSAC sample with an approximately $25 \%$ increase. For both of these samples, decrease is aliphatic carbon is detected, while the amount of CxOy species stays almost unchanged.

The total platinum content detected by XPS, as detailed in Table 2, increased for all four samples. Due to surface sensitivity of XPS sampling depth, this result might point towards significant segregation of Pt towards top layers upon heat treatment. Except HSAC HT which had the least surface platinum content, similar final platinum percentages were found for all samples.

TABLE 2. Platinum Surface Content Comparison as Calculated from XPS

\begin{tabular}{cccc}
\hline Sample ID & Pt \% BOL & Pt \% HT & Pt\% increase \\
\hline LSAC Pt 50 & 4.3 & 6.6 & $54 \%$ \\
MSAC Pt 50 & 2.8 & 7.5 & $169 \%$ \\
HSAC1 Pt50 & 2.5 & 6.1 & $147 \%$ \\
HSAC1 Pt50 HT & 1.9 & 4.7 & $150 \%$ \\
\hline
\end{tabular}

Table 3 shows results of curve fitting Pt $4 \mathrm{f}$ spectra for BOL and heat treated samples.

TABLE 3. Pt Speciation as Determined from XPS Spectra

\begin{tabular}{cccc}
\hline Sample ID & Pt & Pt/Pt-CxHy & PtO/ Pt(OH)2 \\
& $\mathbf{7 1 . 1}$ & $\mathbf{7 1 . 7}$ & $\mathbf{7 2 . 8}$ \\
\hline & Beginning of Life & & 23.9 \\
LSAC 50 & 30.4 & 45.7 & 30.2 \\
MSAC 50 & 33.5 & 36.2 & 25.1 \\
HSAC1 50 & 40.9 & 34.0 & 8.1 \\
HSAC HT 50 & 59.1 & 32.8 & 33.2 \\
LSAC 50 & After Heat Treatment & & 31.2 \\
MSAC 50 & 40.0 & 26.8 & 36.6 \\
HSAC1 50 & 42.0 & 26.8 & 29.0 \\
HSAC HT 50 & 41.3 & 22.0 & 23.7 \\
\hline
\end{tabular}

In BOL samples, increase of surface area causes increase in relative amount of metallic Pt. Heat treating of HSAC sample causes even larger degree of Pt reduction. After heat treatment, all catalyst samples result in very similar composition with approximately $40 \%$ of metallic Pt. The changes introduced into Pt by heat treatment are anti-correlated with increasing carbon surface area.

XPS shows that for LSAC, heat treatment introduces no changes to carbon composition, but significantly increases relative metallic platinum content. MSAC displayed large changes in both carbon and Pt compositions. HSAC saw no changes in 
metallic Pt percentage, but very significant changes in carbon are detected. HSAC HT had the smallest changes of all for both platinum and carbon.

\section{HREELS results}

From the higher energy in the HREELS spectrum, excitations arising from transitions of inner-shell electrons to unoccupied states above the Fermi level can be obtained. For carbon, K-edge spectrum represents the transition from the $1 \mathrm{~s}$ core level to the unoccupied anti-bonding state in the $2 p$ energy level, $\sigma^{*}$ and $\pi^{*}$. Direct measure of $\sigma^{*}$ and $\pi^{*}$ states of carbonaceous materials allows accurate measurement of $\mathrm{sp}^{2}$ and $\mathrm{sp}^{3}$ states provided a suitable reference material with $100 \% \mathrm{sp}^{2}$-hybridization. For both unknown and reference materials, HRELLS K-edge spectra can be used to calculate respective integrated areas under each of energy loss peak at K-edge. Using equation 1, the $\mathrm{sp}^{2}$ fraction can be calculated:

$$
s p^{2} \%=\frac{A_{\pi} *}{A_{\pi} *+\sigma^{*}} / \frac{R_{\pi} *}{R_{\pi} *+\sigma^{*}}
$$

where A - sample, and R- $100 \% \mathrm{sp}^{2}$ bonded reference material, the subscripts $\pi^{*}$ and $\left(\sigma^{*}+\pi^{*}\right)$ represent integrated areas under each energy loss peak at $\mathrm{K}$ edge determined by curve fitting procedure as described below. $(5,6)$

HOPG was used as standard for $100 \% \mathrm{sp}^{2}$ hybridized reference material. The spectra were curve fitted with three Gaussian curves to determine areas under the peaks used for quantifying the ratio of $\mathrm{sp}^{2}$ to $\mathrm{sp}^{3}$ bonds. Figure 2 shows HREELS spectra for HOPG and LSAC 50 sample with three peaks used for curve fitting. The $\pi^{*}$ feature is present at energy loss of $\sim 285 \mathrm{eV}$ which represents transition of $\mathrm{C} 1 \mathrm{~s}$ electrons to unoccupied $\pi^{*}$ state, while the $\sigma^{*}$ feature at $291 \mathrm{eV}$ reflects transition to unoccupied $\sigma^{*}$ state. The $\pi^{*}$ feature is typical for a $\mathrm{sp}^{2}$-hybridized carbon. The $\%$ of $\mathrm{sp}^{2}$-bonded carbon then can be calculated using equation (1).
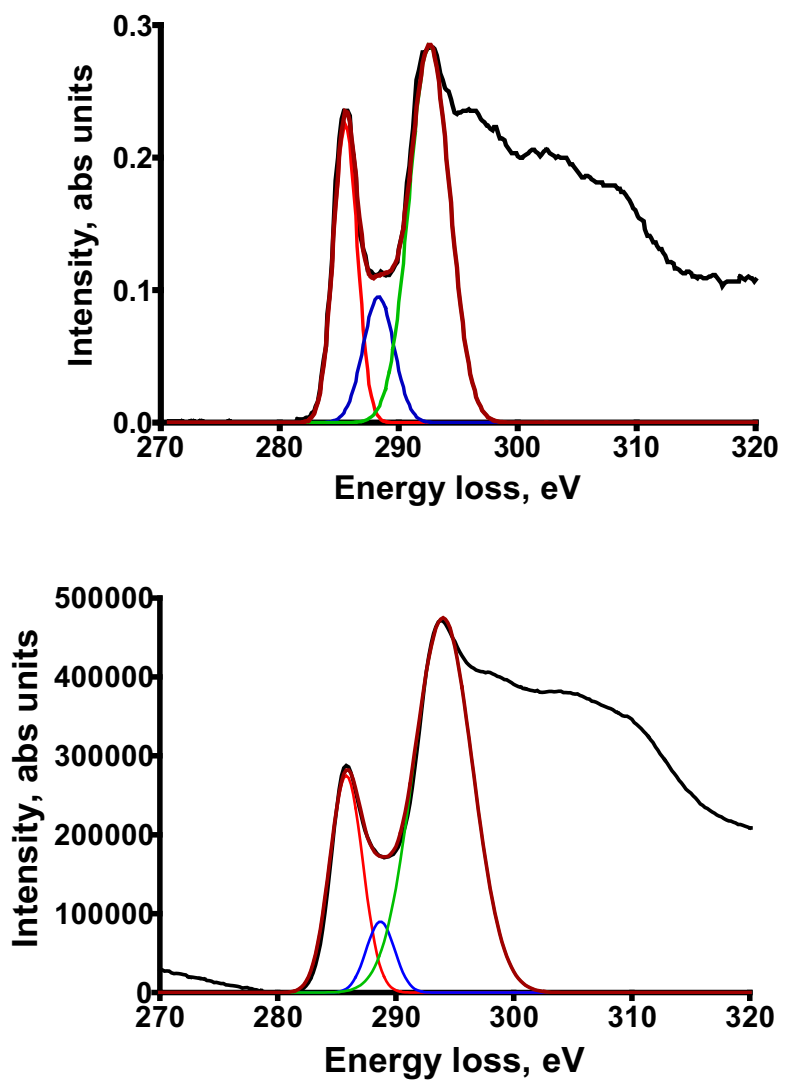

Figure 2. HREELS spectra for a) HPOG and b) LSAC50 samples shown with curve fits used to deconvolve $\sigma^{*}+\pi^{*}$ contributions 
Table 4 shows the percent of $\mathrm{sp}^{2}$ carbon determined from HREELS spectra for 4 samples at beginning of life (BOL) and after ex situ HT treatment at $150^{\circ} \mathrm{C}$ in air $100 \%$ humidity. Beginning of life catalysts have very different levels of graphitic content with highest for LSAC and HSAC HT samples at $\sim 80 \%$. With increasing surface area, graphitic content decreases, with $60 \%$ for HSAC. Heat treatment of samples at $150{ }^{\circ} \mathrm{C}$ in $100 \%$ relative humidity results in samples with quite similar level of graphite content ranging between 77 to $85 \%$. The trend in relative graphitic content for HT samples from sample to sample is the same as for BOL samples, but the range of it is smaller for HT samples.

TABLE 4. $\mathrm{sp} 2 \%$ Determined from HREELS Spectra

\begin{tabular}{cccc}
\hline Sample ID & BOL & HT & \% Increase \\
\hline LSAC 50 & $78.7 \%$ & $85.0 \%$ & $8.0 \%$ \\
MSAC 50 & $69.7 \%$ & $79.2 \%$ & $13.6 \%$ \\
HSAC 50 & $59.4 \%$ & $77.3 \%$ & $30.0 \%$ \\
HSAC HT 50 & $80.2 \%$ & $83.6 \%$ & $4.2 \%$ \\
\hline
\end{tabular}

The trend in change of graphitic content determined by HREELS is very similar to that detected by XPS, except for MSAC sample having smaller relative $\%$ of graphitic carbon as detected by XPS then HSAC sample. This might be due to surface sensitivity of XPS in comparison with HREELS, pointing towards larger relative amounts of surface carbon oxides.

Figure 3 plots percent of graphitic content determined by XPS vs. that determined by HREELS for both BOL and HT samples. Very good correlation is observed for both sets of samples. For BOL samples, smaller spread of values detected by XPS indicates that surface is different from the bulk and surfaces of materials are more similar to each other than the bulk of catalysts. HT samples are very similar as detected by both methods, but similarity is larger at the very top $10 \mathrm{~nm}$ as seen by XPS. The graphitic carbon content of all samples after HT is similar to that of LSAC and HSAC HT samples.

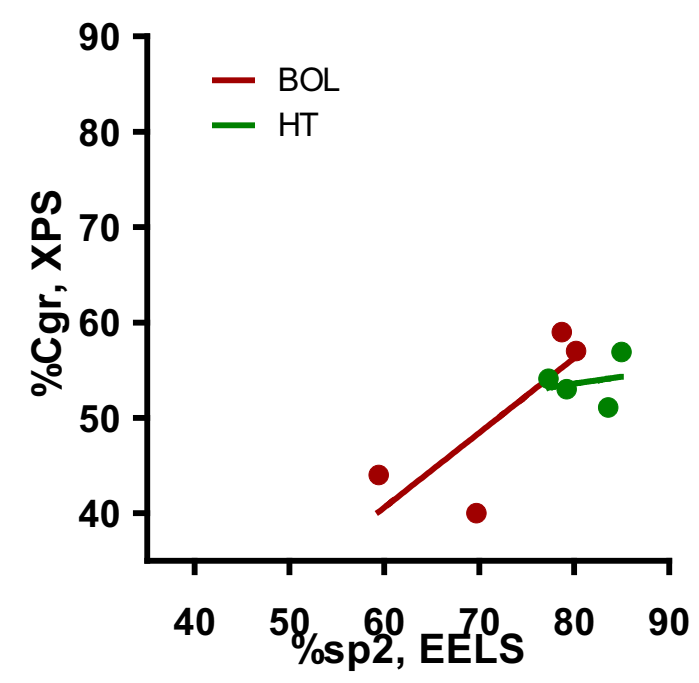

Figure 3. Percent graphitic carbon as determined by XPS vs \%sp2 content as determined by HREELS

\section{$\underline{\mathrm{RDE} \text { results }}$}

Catalyst performance losses were quantified using rotating disk electrode experiments. Two sets of catalysts were tested for this study: unaltered catalysts and aged catalysts. Catalysts were aged by heating the unaltered samples for two hours at $150^{\circ} \mathrm{C}$ and $100 \%$ RH. A portion of the sample was then weighed and was added to a solution of $80 / 20$ DI water to isopropyl alcohol and a $0.5 \%$ Nafion in DI water. The mixture was then sonicated to form a homogenous solution. In the end, a $4 \mathrm{mg} / \mathrm{ml}$ ink with $30 \mu \mathrm{l}$ of the Nafion solution per milligram of sample was made. To test the samples, $10 \mu \mathrm{l}$ of the catalyst ink were pipetted on to a rotating disk electrode, and was then dried for 30 minutes. 
Using a rotation speed of 1600 RPM and $0.1 \mathrm{M}$ perchloric acid as the electrolyte, the electrode was cycled between 0 and $1.2 \mathrm{~V}$ (vs. RHE) 50 times at a rate of $100 \mathrm{mV} / \mathrm{s}$. This step was done to ensure a clean, uncontaminated surface. After the cleaning sweeps, oxygen gas was bubbled through the cell, and the electrode was cycled between 0 and 1.2 $\mathrm{V}$ (vs. RHE) at a rate of $5 \mathrm{mV} / \mathrm{s}$. The exchange current densities and Tafel slopes for ORR were then extracted from these curves to quantify performance losses due to aging. The exchange current density and Tafel slopes were calculated by plotting the first 100 $\mathrm{mV}$ of the cathodic sweep below the open circuit potential on a semi-log plot and fitting a linear trendline to the curve. The slope of the trendline would be equivalent to the Tafel slope, while the intercept would be equal to the exchange current density. The date obtained can be seen in Tables 5 and 6 below.

Table 5. Tafel Slopes (mV/decade)

\begin{tabular}{lccc}
\hline Sample ID & BOL & After Heat Treatment & \% Change \\
\hline LSAC 50 & 27.2 & 21.5 & -20.8 \\
MSAC 50 & 30.6 & 27.5 & -10.3 \\
HSAC1 50 & 32.8 & 29.1 & -11.3 \\
HSAC HT 50 & 24.0 & 28.3 & +18.0 \\
\hline
\end{tabular}

Table 6. Exchange Current Densities ( $\mathrm{mA} / \mathrm{cm} 2)$

\begin{tabular}{lccc}
\hline Sample ID & BOL & After Heat Treatment & \% Change \\
\hline LSAC 50 & 4.2 & 2.9 & -32.2 \\
MSAC 50 & 3.8 & 3.2 & -17.2 \\
HSAC1 50 & 4.0 & 3.9 & -1.6 \\
HSAC HT 50 & 3.1 & 3.4 & +9.9 \\
\hline
\end{tabular}

The data shows that through the ex situ aging, the performance of all catalysts are changed, but not uniformly. While XPS and HREELS data showed that the aging process increased the relative graphite content for each sample, ex situ aging generally decreased the overall performance of the catalysts. While seeing decreased performance due to aging is a well-documented phenomenon, seeing an increase in the Tafel slope and exchange current density of HSAC HT is unexpected. (7-9) Also surprising is that, after excluding HSAC HT, the loss of performance is correlated with increase in graphitic content as detected by both XPS and HREELS, as seen in Figure 4. LSAC which has the highest graphite content after heat treatment, has the largest loss in performance. Furthermore, HSAC had the lowest percentage of graphite, yet it suffered the lowest performance losses.
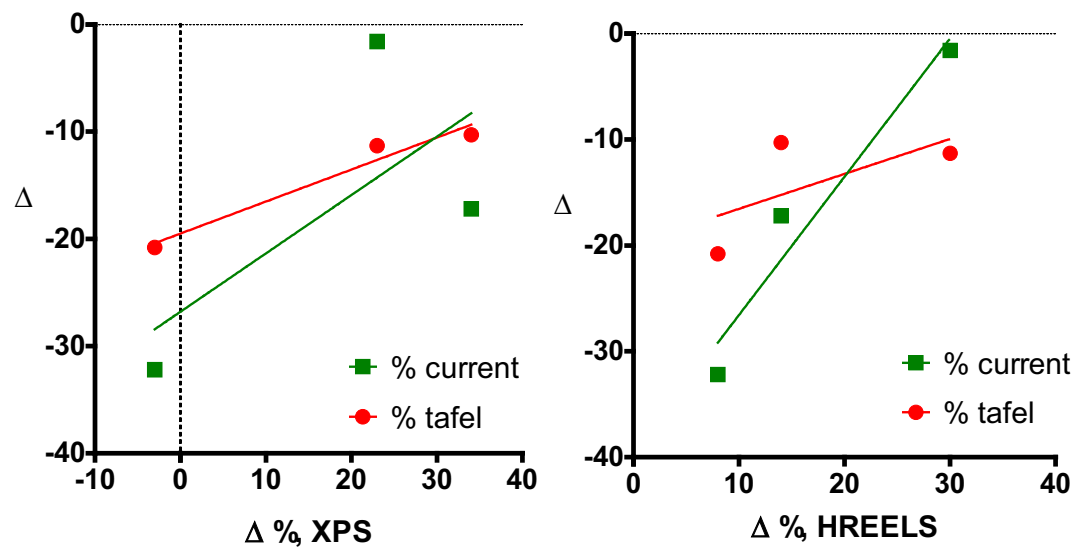

Figure 4. Change in Tafel plot and Exchange Current Densities plotted versus change in graphitic content as detected by XPS (left) and HREELS (right) excluding HSAC HT results

Figure 5 plots the changes in relative and absolute percentages to metallic $\mathrm{Pt}$ versus change in Tafel plot. Very different behavior for these two ways to represent XPS data are observed. Anti-correlation between changes in Tafel slope and change in relative amount of metallic $\mathrm{Pt}$ and correlation between change in absolute amount of metallic $\mathrm{Pt}$ and change in Tafel slope are detected by XPS. Relative \% do not take into account overall increase due to segregation of $\mathrm{Pt}$, so correlation of losses in performance with 
absolute \% of metallic $\mathrm{Pt}$ is more adequate. For larger degree of Pt reduction smaller degree of losses are observed.

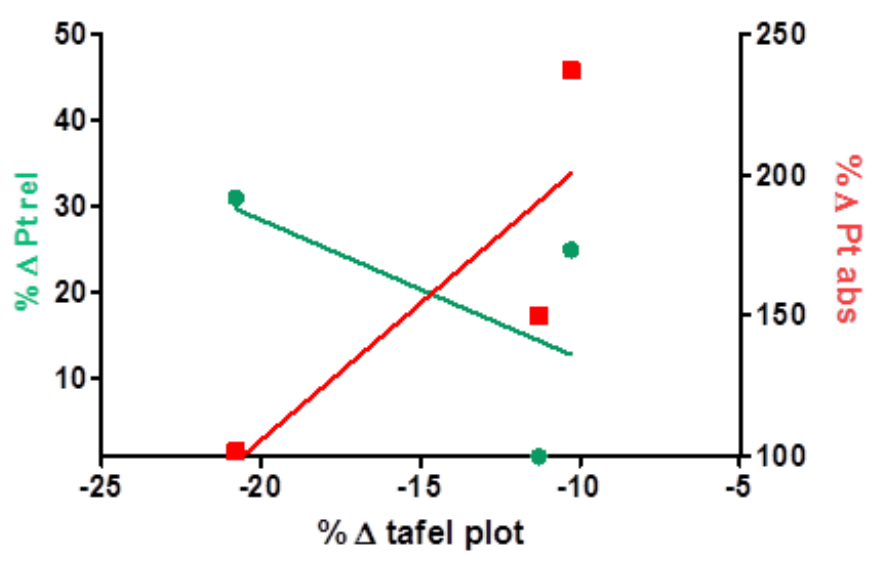

Figure 5. Changes in Tafel slopes vs. relative and absolute platinum content on the surface

Though the electrochemical data would suggest that higher losses are observed with more graphitic samples and less reduced Pt, the actual dependence between changes induced by heating and performance is more complicated. Because HSAC HT had already been heat treated before it was analyzed, it had already lost a significant portion of the amorphous content found in BOL HSAC. As such, any performance loss due to platinum dissolution or islanding caused by corroding the amorphous components would not be seen in its initial performance. Also, after further heat treatment, both the catalyst performance and the graphite content were seen to increase. This could indicate that the losses seen after heat treatment are not due increasing graphitic carbon content, but potentially the loss of bulk platinum associated with the amorphous content. Finally, while HSAC did have the smallest performance losses, but it also had the largest gain in graphite content, making it chemically more similar to HSAC HT than it was originally. It is possible that further heat treatment of the samples would lead to increases in performance instead of losses.

\section{Conclusions}

Ex situ heat treatment of four carbon supported platinum catalysts was performed in order to determine the effect graphite content has on catalyst performance. The samples were analyzed using XPS, HREELS, and RDE experiments. Though both XPS and HREELS determined that graphite and metallic platinum content increased due to the heat treatment, the increases were not homogenous, and that the surface of the samples differed from the bulk. In addition, it was shown that as prepared samples displayed decreased performance after heat treatment, while a previously heat treated sample had increased catalytic activity. Current speculation suggests that the losses seen were not due increased graphite content, but loss of platinum previously bound to corroded amorphous carbon.

\section{Acknowledgments}

The authors acknowledge the financial assistance from the Department of Energy EERE Hydrogen and Fuel Cell Technology Program (Project DE-EE0000466) and Natural Resources Canada (Project 414-CETC-526)

\section{References}

1. F. Dundar, A. Smirnova, X. Dong, A. Ata and N. Sammes, Journal of Fuel Cell Science and Technology, 3, 428 (2006).

2. T. J. Schmidt, H. A. Gasteiger and R. J. Behm, Journal of The Electrochemical 
Society, 146, 1296 (1999).

3. J. O. Muller, D. S. Su, U. Wild and R. Schlogl, Physical Chemistry Chemical Physics, 9, 4018 (2007).

4. A. Patel, K. Artyushkova, P. Atanassov, A. P. Young, M. Dutta, Z. Ahmad, V. Colbow, S. Wessel and S. Ye, ECS Transactions, 33, 425 (2010).

5. M. Knauer, M. E. Schuster, D. Su, R. Schlögl, R. Niessner and N. P. Ivleva, The Journal of Physical Chemistry A, 113, 13871 (2009).

6. S. R. P. Silva and V. Stolojan, Thin Solid Films, 488, 283 (2005).

7. D. A. Stevens, M. T. Hicks, G. M. Haugen and J. R. Dahn, Journal of The Electrochemical Society, 152, A2309 (2005).

8. C. G. Chung, L. Kim, Y. W. Sung, J. Lee and J. S. Chung, International Journal of Hydrogen Energy, 34, 8974 (2009).

9. H. Chizawa, Y. Ogami, H. Naka, A. Matsunaga, N. Aoki, T. Aoki and K. Tanaka, ECS Transactions, 11, 981 (2007). 